Physics International 1 (1): 22-26, 2010

ISSN 1948-9803

(C) 2010 Science Publications

\title{
The Effects of Fatigue and Chronic Ankle Instability on Dynamic Postural Control
}

\author{
Seyed Hossein Hosseinimehr, Hassan Daneshmandi and Ali Asghar Norasteh \\ Department of Sport Medicine and Corrective Exercise, \\ Guilan University, P.O. Box 41635-4113, Rasht, Iran
}

\begin{abstract}
Problem statement: The proprioception information is a prerequisite for balance, body's navigation system and the movement coordinator. The aim of this study was to investigate the effects of fatigue and chronic ankle instability on dynamic postural control. Approach: Thirty physical education students ( 14 males, 16 females) in two groups ( 15 healthy age $=21.78 \pm 0.79$ years, height $=168.3 \pm 5.6 \mathrm{~cm}$ and weight $=63.6 \pm 2.6 \mathrm{~kg}, 15$ with chronic ankle instability age $=21.43 \pm 0.83$ years, height $=168.4 \pm 5.5 \mathrm{~cm}$ and weight $=63.3 \pm 3.2 \mathrm{~kg}$ ) participated in this study voluntary. Star Excursion Balance Test was used for evaluation of dynamic postural control. Subjects performed a functional fatigued protocol that lasted $15 \mathrm{~min}$. Using the Borg scale, also measured rating of perceived exertion before, middle and after the fatigue protocol. Results: The findings indicated that dynamic postural control decreased after fatigue in two groups $(\mathrm{p}<0.05)$. In contrast, there was only significance different between two groups after fatigue in lateral and antrolateral directions of Star Excursion Balance Test $(\mathrm{p}<0.05)$. Conclusion: Fatigue and chronic ankle instability can effect on dynamic postural control also, combination fatigue and chronic ankle instability can increase this effect. High incidence of ankle sprains especially in lateral and antrolateral directions which lead to reducing of balance can be practical guideline for training programs and management of fatigue in athletes. This finding also helps to sport physiotherapists to more emphasis on ankle sprain in rehabilitations on lateral and antrolateral directions.
\end{abstract}

Key words: Dynamic postural control, Star excursion balance test, fatigue, chronic ankle instability

\section{INTRODUCTION}

Lateral ankle sprains are one of the most common injuries among athletes (Garrick and Requa, 1988). After initial injury, the rate of recurrence may be as high as 80\% among athletes (Yeung et al., 1994). Altered mechanical joint stability due to repeated disruptions to ankle integrity with resultant perceived and observed deficits in neuromuscular control has been described as Chronic Ankle Instability (CAI) (Beynnon et al., 2002). Aspects of neuromuscular control may be quantified through measures of postural control. Postural control can be defined as either static (maintaining a position with minimal movement), semidynamic (maintaining a position while the base of support moves), or dynamic (maintaining a stable base of support while completing a prescribed movement) (Winter et al., 1990).

The Muscular Fatigue (MF) that is defined as any reduction in the neuromuscular ability to produce strength (Rozzi et al., 2000) is a usual phenomenon in resistance sports and is a common experience in the daily activities (Johnston et al., 1998). The beginning of the voluntary muscular activity involves several processes that start with the cortical control in the brain and end with the cross-bridges inside the muscular fiber. Therefore, the muscular fatigue can be the result of a failure in any process involved in the muscular contraction (Johnston et al., 1998). Until recently, most research completed on proprioception and the lower extremity examined balance or joint position sense (Nakagawa and Hoffman, 2004). These studies often examined proprioception of the ankle and/or knee during an injured state, or evaluated the benefits of balance training when rehabilitating an injury (Nakagawa and Hoffman, 2004). More recently, fatigue has become a popular focus of research, examining fatigue and the relationship to ankle sprains (Gribble and Hertel, 2004; Huston et al., 2005; Nakagawa and Hoffman, 2004) and knee injuries (Hiemstra et al., 2001; Rowe et al., 1999; Skinner et al., 1986) with regard to altered postural control. Gribble and Hertel

Corresponding Author: Seyed Hossein Hosseinimehr, Department of Science in Sport Medicine and Corrective Exercise, University of Guilan, P.O. Box 41635-4113, Rasht, Iran Tel: 0098-9173034237 
(2004) examined this further as they conducted a study on how fatiguing various muscles of the lower extremity (flexors and extensors of the hip and knee and dorsiflexors and plantarflexors of subtalar joint) affected static postural stability as measured by a static unilateral leg stance. They found that fatiguing muscles more proximally altered static postural control more than fatiguing the more distal muscles (Gribble and Hertel, 2004). Other problem with the current research is that it often uses a repeated isokinetic exercise, or an isometric hold to fatigue the muscle or muscles being examined. Although an easy standardized way to fatigue a muscle or muscle group, these activities are usually not functional or sport related in anyway. However, there are few times in any sport where a player will be standing in a static isometric plantarflexed position until they can not hold it anymore and then play in a game. Since researchers have been able to develop this vast base of support knowledge in this area, it is time to take the next step. Research needs to support a more functional or sportrelated fatiguing protocol, to observe how the rigors of athletic competition affects the dynamic postural control system of the body. There is a need to determine if the coordinated movements involved in sports along with cardiovascular endurance combine to produce a different level of fatigue that can have a detrimental effect on dynamic postural control. It appears that with the lack of current research linking dynamic postural control and fatigue and the current concepts in rehabilitation that one would want to examine the possible connection between overall body fatigue and how it interacts with dynamic postural control. Also, a majority of these fatiguing studies have evaluated static postural control (Gribble and Hertel, 2004; Huston et al., 2005; Nakagawa and Hoffman, 2004) or joint position sense at the ankle with or without a fatiguing protocol (Hiemstra et al., 2001; Rowe et al., 1999; Skinner et al., 1986). Therefore the purpose of this study is to examine the effects of an activity related fatiguing protocol fatigue and chronic ankle instability on dynamic postural control.

\section{MATERIALS AND METHODS}

Participants: Thirty physical education students from Guilan University volunteered as subjects. Individuals with self-reported vestibular disorders or mild head injury in the previous 6 months were excluded from the study. After completing the consent form, subjects were placed into 1 of 2 groups: healthy ( 7 males, 8 females, age $=21.78 \pm 0.79$ years, height $=168.3 \pm 5.6 \mathrm{~cm}$ and weight $=63.6 \pm 2.6 \mathrm{~kg})$ and CAI $(7$ males, 8 females, age $=21.43 \pm 0.83$ years, height $=168.4 \pm 5.5 \mathrm{~cm}$ and weight $=63.3 \pm 3.2 \mathrm{~kg}$ ). The healthy subjects were free of any self-reported lower extremity injury in the previous 6 months. Subjects in the CAI group were free from injury to the lower extremity other than the ankle in the previous 6 months; had a history of at least 1 acute ankle sprain that resulted in swelling, pain and temporary loss of function (but none in the previous 3 months); and a history of multiple episodes of the ankle "giving way" in the past 6 months.

\section{Instruments:}

Star excursion balance test: This is a test that incorporates a single-leg stance on one leg with maximum reach of the opposite leg. The test is consisted of 8 lines that make a $45^{\circ}$ angle to one another. The $45^{\circ}$ increments are from the center of the grid. The 8 lines positioned on the grid are labeled according to the direction of excursion relative to the stance leg (anterior, anterolateral, anteromedial, medial, lateral, posterior, posterolateral, posteromedial) (Kinzey and Armstrong, 1998). The diameter of the circle is $182 / 9 \mathrm{~cm}$ and it is placed on a firm surface. The width of each line is $7 / 62 \mathrm{~cm}$. In order to reduce the learning effect each subject choose 6 directions out of the 8 to practice (Blackburn et al., 2000). The subject stood in the middle of the circle with the dominant leg; then with the opposite leg he reached for the furthest marked distance (Fig. 1). Each subject was asked to touch the furthest part of the line with the most distal part of his reach foot. This was done with control and in a slow manner to ensure adequate neuromuscular control of the stance leg. The subject then returned to the original stance and the touch points that were marked during examination were recorded.

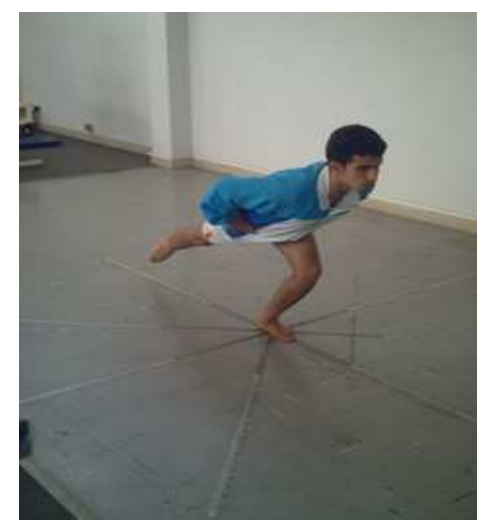

Fig. 1: A subject performing the posterior-reach component of the star excursion balance tests 
Three second rest were allocated between each reach. The direction of the revolution based on the right or left reach legs was clock wise and counter clock wise, respectively (Blackburn et al., 2000). The reach was not accepted if the leg could not touch the target line, if the subject's weight was shifted to the reach leg, if the support leg was lifted from the center, or if balance was disturbed during the reach (Blackburn et al., 2000).

Fatigue protocol: The fatiguing protocol consisted of seven stations each designed to fatigue the subject by using different energy systems to represent a sportrelated fatigue. Each station was timed by Triathlon watch. Just prior to the first station, the principal investigator took the Rating of Perceived Exertion (RPE) (Winter et al., 1990) reading from the participant.

Station one consisted of a 5 min moderate Jog at the subjects self-selected pace. This jog took place around the perimeter of the gym in order to help the participant maintain a steady pace. Station two consisted of 2 min of sprints up and down the length of a basketball court. Station three was 1 min of push-ups. After station three was completed, the RPE measurement was taken as station three is considered the half-way point. Station four consisted of $1.5 \mathrm{~min}$ of sit-ups. Station five was $2 \mathrm{~min}$ of 12 inch step-ups. Station six consisted of another 2 min of sprints up and down the basketball court and station seven was 1.5 min run at as fast a pace as the participant could maintain for the entire $1.5 \mathrm{~min}$ (Susco et al., 2004; Wilkins et al., 2004). A third RPE was recorded as soon as the subject completed the last station. Adequate fatigue has been deemed to be an RPE of 15 which represents $75-90 \%$ maximum oxygen consumption (Susco et al., 2004; Wilkins et al., 2004) immediately after taking the RPE; the subject completed the post-test (post-fatigue) Star Excursion Balance Test. The procedure was similar as the pre-test with the only exception being the starting excursion was again chosen at random by the principal investigator.

Statistically analyses: A one-way repeated measures ANOVA was used to determine if a difference existed for Rating of Perceived Exertion. Post-hoc testing consisted of three pairwise comparisons (pre-test to mid-test, pre-test to post-test and mid-test to post-test) to determine which changes were significant. Paired sample test was used for comparison of difference between pre and post test, Independent sample test was used for comparison of difference between two groups in dynamic postural control. The $\mathrm{p}$ value for all tests was set at $\mathrm{p}<0.05$.

\section{RESULTS}

Repeated measured $(1 \times 3)$ ANOVA showed there was significant difference for RPE (for control group, $\mathrm{F}_{1,14}=3988.31, \mathrm{p}<0.001$, for experiment group $\left.\mathrm{F}_{1,14}=4355.34, \mathrm{p}<0.001\right)$. also post-hoc (LSD) showed that pre-test to mid-test $(\mathrm{p}<0.001)$, pre-test to post-test $(\mathrm{p}<0.001)$ and mid-test to post-test $(\mathrm{p}<0.001)$ were all statistically significant in two groups (Fig. 2). Paired sample test showed there was significant difference between mean of reached distance $(\mathrm{cm})$ in pre and post test in 8 directions of star excursion balance test in two groups (Fig. 3 and $4, \mathrm{p}<0.05$ ). In contrast, there was significance different between two groups after fatigue in lateral and antrolateral directions in dynamic postural control only $(\mathrm{p}<0.05)$ (Table 1$)$.

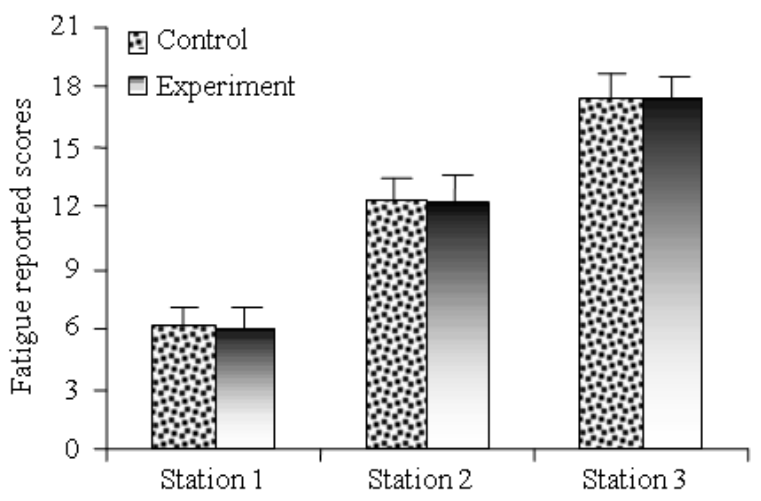

Fig. 2: Difference of fatigue reported scores in two groups

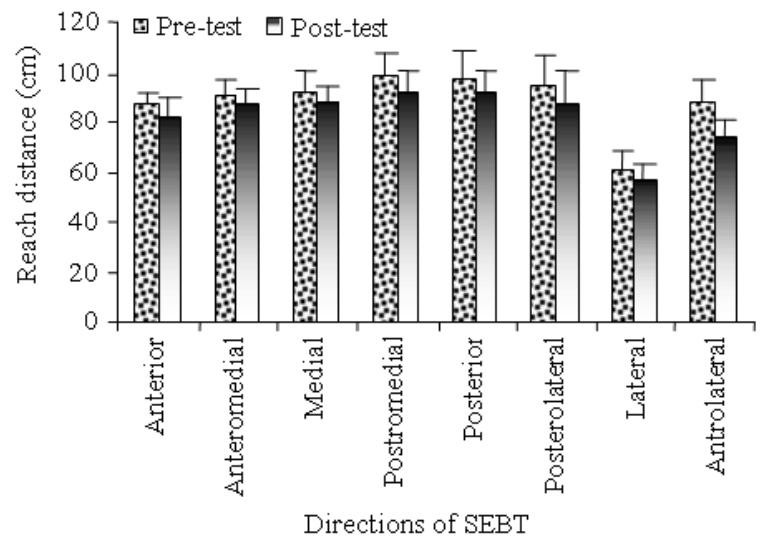

Fig. 3: The means comparison of the pre and post test for the distance in the eight directions of the dynamic balance in control group 
Phy. Intl. 1 (1): 22-26, 2010

Table 1: The results for the $t$ test correlation with respect to the reached distance in $\mathrm{cm}$ between two groups

\begin{tabular}{|c|c|c|c|c|c|}
\hline \multirow{2}{*}{$\begin{array}{l}\text { Directions } \\
\text { of SEBT }\end{array}$} & \multirow[b]{2}{*}{ Group } & \multicolumn{2}{|l|}{ Mean \pm SD } & \multirow[b]{2}{*}{$\mathrm{t}$} & \multirow[b]{2}{*}{$\mathrm{P}$} \\
\hline & & Pre-test & Post-test & & \\
\hline \multirow{3}{*}{ Anterior } & Control & $87.78 \pm 4.8$ & $82.80 \pm 6.6$ & \multirow{3}{*}{1.12} & \multirow{3}{*}{0.21} \\
\hline & & & & & \\
\hline & Experiment & $89.80 \pm 7.4$ & $81.90 \pm 6.4$ & & \\
\hline \multirow{3}{*}{ Antero medial } & Control & $91.34 \pm 5.4$ & $87.00 \pm 6.1$ & \multirow{3}{*}{-0.92} & \multirow{3}{*}{0.36} \\
\hline & & & & & \\
\hline & Experiment & $91.85 \pm 8.9$ & $85.33 \pm 4.4$ & & \\
\hline \multirow{3}{*}{ Medial } & Control & $92.27 \pm 7.9$ & $85.33 \pm 4.4$ & \multirow{3}{*}{-1.20} & \multirow{3}{*}{0.23} \\
\hline & & & & & \\
\hline & Experiment & $93.17 \pm 9.9$ & $86.6 \pm 5.2$ & & \\
\hline \multirow{3}{*}{ Postero medial } & Control & $99.90 \pm 8.5$ & $92.30 \pm 7.8$ & \multirow{3}{*}{-0.98} & \multirow{3}{*}{0.33} \\
\hline & & & & & \\
\hline & Experiment & $94.27 \pm 11.46$ & $92.30 \pm 7.8$ & & \\
\hline \multirow{3}{*}{ Posterior } & Control & $97.10 \pm 11.6$ & $91.76 \pm 8.7$ & \multirow{3}{*}{0.97} & \multirow{3}{*}{0.33} \\
\hline & & & & & \\
\hline & Experiment & $91.76 \pm 9.7$ & $84.26 \pm 7.6$ & & \\
\hline \multirow{4}{*}{ Postero lateral } & Control & $94.78 \pm 12.6$ & $82.16 \pm 9.5$ & \multirow{3}{*}{1.20} & \multirow{3}{*}{0.22} \\
\hline & & & & & \\
\hline & Experiment & $88.44 \pm 6.6$ & $82.16 \pm 9.5$ & & \\
\hline & Control & $60.88 \pm 5.5$ & $56.70 \pm 7.6$ & \multirow{3}{*}{-2.40} & \multirow{3}{*}{$0.01 *$} \\
\hline \multirow[t]{3}{*}{ Lateral } & & & & & \\
\hline & Experiment & $61.44 \pm 7.5$ & $50.66 \pm 6.03$ & & \\
\hline & Control & $88.46 \pm 8.6$ & $73.76 \pm 7.6$ & \multirow{3}{*}{-3.60} & \multirow{3}{*}{$0.001^{*}$} \\
\hline \multirow[t]{2}{*}{ Antero lateral } & & & & & \\
\hline & Experiment & $84.76 \pm 6.4$ & $75.25 \pm 5.2$ & & \\
\hline
\end{tabular}

*: Significant differences

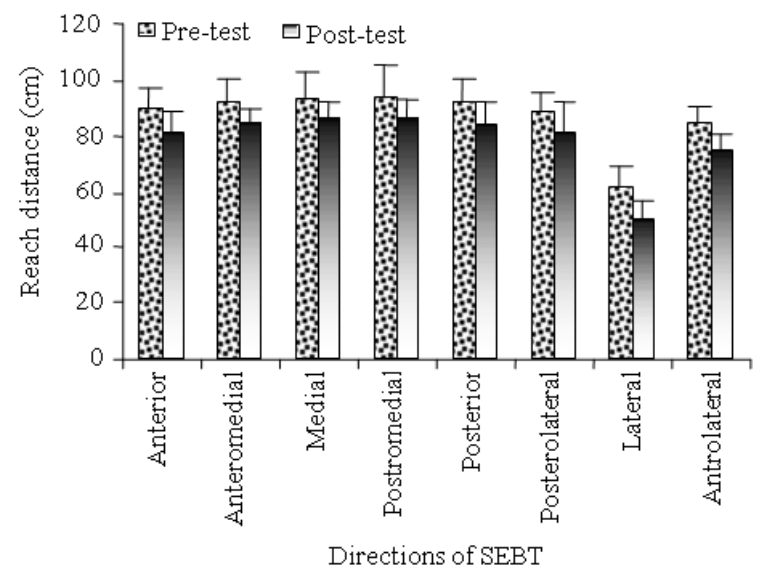

Fig. 4: The means comparison of the pre and post test for the distance in the eight directions of the dynamic balance in experiment group

\section{DISCUSSION}

To begin the discussion, it must be known that this study is the first to report on an activity related fatiguing protocol and chronic ankle instability on dynamic postural control. A majority of other fatiguing studies have evaluated static postural control (Gribble and Hertel, 2004; Huston et al., 2005; Nakagawa and Hoffman, 2004) or joint position sense at the ankle with or without a fatiguing protocol (Hiemstra et al., 2001; Rowe et al., 1999; Skinner et al., 1986). These studies that used a local fatiguing protocol followed either an isokinetic fatiguing protocol, a repeated lifting protocol, or an isometric fatiguing protocol. Few studies, if any exist in the literature that use a central or general fatigue protocol to evaluate dynamic postural control especially in athletes with chronic ankle instability. Lepers et al. (2002) used a cycling bout protocol, but did not examine dynamic postural control (Susco et al., 2004). Susco et al. (2004) and Wilkins et al. (2004) used this functional fatiguing protocol, but evaluated the subject's performance on the Balance Error Scoring System (BESS), a measure of static balance. Although recently there has been extensive research on fatigue and dynamic postural control as measured by the Star Excursion Balance Test (SEBT), there is limited research available examining a sport-related fatiguing process and the effects it would have on dynamic postural control especially in athletes with chronic ankle instability? An activity related fatiguing process would involve an exercise bout that would more closely resemble a "typical" practice or game and would fatigue both local and central processes instead of just concentrating on a single muscle or muscle group. This fatiguing process would include various types of exercise in an attempt to fatigue the athlete including but not limited to isokinetic exercise, isotonic exercise, plyometric and agility exercises, as well as testing their cardiovascular endurance. One of the factors in developing and designing a central or general fatiguing protocol is that it actually is taxing to all energy systems. Although fatigue substrates and metabolites were not determined in this study or the Wilkins et al. (2004) or Susco et al. (2004) studies, Susco et al. (2004) determined that an activity of this nature does cause fatigue and that fatigue lasts at least $20 \mathrm{~min}$. The posttest SEBT took on average 10 min to complete, with the longest time being $13 \mathrm{~min}$. If one considers the $20 \mathrm{~min}$ fatigue time span in the Susco et al. (2004) study, the participants should still have been fatigued and were still reporting feelings of fatigue. To ensure fatigue occurred in all three studies, the rating of perceived exertion was used to determine the participant's level of fatigue.

Proprioception is the specialized variation of the sensory modality of touch that encompasses the sensation of joint movement. The application of proprioception requires afferent information from the many mechanoreceptors like the muscle spindles, Golgi tendon organs, Ruffini endings, Pacinian Corpuscles 
and cutaneous receptors. Proprioception requires the peripheral nervous system to house the mechanoreceptors and send signals to the spinal cord. The central nervous system, which consists of the brain and spinal cord, sends signals from the motor cortex, down the descending tracts and out to the muscles via motor neurons. Neuromuscular control is the efferent response to the afferent stimuli presented by the mechanoreceptors. It is represented by the muscle movement in response to the muscle stretch signaled by the muscle spindle (Nicolas and Nicolas, 2005). In comparison of two groups after fatigue protocol in this study, may be because created lateral ankle sprain and follow up decrease flexibility, rang of motion and strength in lateral ankle in athletes with chronic ankle instability, result in decrease of distance reach in lateral and antrolateral directions in star excursion balance test.

\section{CONCLUSION}

Although it is not realistic to expect a coach to stop practice or decrease practice times because someone is experiencing fatigue which may lead to injury, this topic needs attention. To be realistic, most coaches could rearrange how practices are conducted. For example, a coach who is having $3 \mathrm{~h}$ basketball practices could schedule the more difficult and higher risk activities in the first half of practice and leave the lower risk activities for the last hour when the athletes will be feeling the effects of fatigue and this problem must care in athletes with chronic ankle instability. On the other hand, high incidence of ankle sprains especially in lateral and antrolateral directions which lead to reducing of balance can be practical guideline for training programs and management of fatigue in athletes. Also, this finding also helps to sport physiotherapists to more emphasis on ankle sprain in rehabilitations on lateral and antrolateral directions.

\section{REFERENCES}

Beynnon, B.D., D.F. Murphy and D.M. Alosa, 2002. Predictive factors for lateral ankle sprains: A literature review. J. Athlet. Train., 37: 376-380.

Blackburn, T., K.M. Guskiewicz, M.A. Petschaur and W.E. Prentice, 2000. Balance and joint stability: The relative contributions of proprioception and muscular strength. J. Sport Rehabil., 9: 315-328.

Garrick, J.G. and R.K. Requa, 1988. The epidemiology of foot and ankle injuries in sports. Clin. J. Sports Med., 7: 29-36.

Gribble, P.A. and J. Hertel, 2004. Effect of lowerextremity muscle fatigue on postural control. Arch. Phys. Med. Rehabil., 85: 589-592.
Hiemstra, L.A., I.K. Lo and P.J. Fowler, 2001. Effect of fatigue on knee proprioception: Implications for dynamic stabilization. J. Orthop. Sports Phys. Ther., 31: 598-605.

Huston, J.L., M.A. Sandrey, M.W. Lively and K. Kotsko, 2005. The effects of calf-muscle fatigue on sagittal-plane joint-position sense in the ankle. J. Sport Rehabil., 14: 168-184.

Johnston, R.B., M.E. Howard, P.W. Cawley and G.M. Lossee, 1998. Effect of lower extremity muscular fatigue on motor control erformance. Med. Sci. Sports Exerc., 30: 1703-1707.

Kinzey, S.J. and C.W. Armstrong, 1998. The reliability of the star-excursion test in assessing dynamic balance. J. Orthop. Sports Phys., 27: 356-360.

Lepers, R., N. Maffiuletti, L. Rochette, J. Brugniaux and F. Millet, 2002. Neuromuscular fatigue during a long-duration cycling exercise. J. Applied Physiol., 92: 1487-1493.

Nakagawa, L. and M. Hoffman, 2004. Performance in static, dynamic and clinical tests of postural control in individuals with recurrent ankle sprains. J. Sport Rehabil., 13: 255-268.

Nicolas, V. and P. Nicolas, 2005. Postural control during quiet standing following cervical muscular fatigue: Effect of changes in sensory inputs. J. Neurosci, 378: 135-139.

Rowe, A., S. Wright, J. Nyland, D.N.M. Caborn and R. Kling, 1999. Effects of a $2 \mathrm{~h}$ cheerleading practice on dynamic postural stability, knee laxity and hamstring extendibility. J. Orthop. Phys. Ther., 29: 455-462.

Rozzi, S., P. Yuktanandana, D. Pincivero and S. Lephart, 2000. Role of Fatigue on Proprioception and Neuromuscular Control. In: Proprioception and Neuromuscular Control in Joint Stability, Lephart, S. and F. Fu (Eds.). Human Kinetics, Champaign, IL., pp: 375-383.

Skinner, H.B., M.P. Wyatt, J.A. Hodgdon, D.W. Conrad and R.L. Barrack, 1986. Effect of fatigue on joint position sense of the knee. J. Orthop. Res., 4: 112-118.

Susco, T.M., T.C.V. McLeod, B.M. Gansneder and S.J. Shultz, 2004. Balance recovers within $20 \mathrm{~min}$ after exertion as measured by the balance error scoring system. J. Athlet. Train., 39: 241-246.

Wilkins, J.C., T.C.V. McLeod, D.H. Perrin and B.M. Gansneder, 2004. Performance on the balance error scoring system decreases after fatigue. J. Athlet. Train., 39: 156-161.

Winter, D.A., A.E. Patla and J.S. Frank, 1990. Assessment of balance control in humans. Med. Prog. Technol., 16: 31-51.

Yeung, M.S., K.M. Chan, C.H. So and W.Y. Wuan, 1994. An epidemiological survey on ankle sprain. Br. J. Sports Med., 28: 112-116. 